\title{
The Kety-Schmidt Technique for Quantitative Perfusion and Oxygen Metabolism Measurements in the MR Imaging Environment
}

\author{
J.J. Lee, W.J. Powers, C.B. Faulkner, P.J. Boyle, and C.P. Derdeyn
}

\begin{abstract}
SUMMARY: The Kety-Schmidt technique provides quantitative measurement of whole-brain CBF. CBF is measured as the area between the arterial and venous washout curves of a diffusible tracer. Oxygen extraction and metabolism may be calculated from arterial and venous samples. In this report, we present a method for performing these measurements in an MR imaging environment. This technique could be useful for validation of MR imaging methods of hemodynamic and metabolic measurements in humans.
\end{abstract}

ABBREVIATIONS: $\mathrm{CMRO}_{2}=$ cerebral metabolic rate for oxygen consumption; $\mathrm{IM}=$ intramuscularly; $\mathrm{NDIR}=$ nondispersive infrared; OEF = oxygen extraction fraction

A ccurate, quantitative, and noninvasive measurements have great potential for advancing the study of human cerebral hemodynamics in health and disease and clinical patient care. MR imaging methods for measuring CBF have yielded results in animals and humans that are consistent with those of PET. ${ }^{1,2}$ However, direct quantitative validation of MR imaging CBF measurements with the criterion standard is still lacking.

The seminal studies of CBF and oxygen metabolism performed by Seymour Kety and Carl Schmidt ${ }^{3}$ and their tracer kinetic methods were the basis for validating PET measurements of CBF by using O-15-labeled water. The method entails placement of jugular venous and arterial catheters. During either wash-in or washout from the equilibrium of a freely diffusible tracer (generally nitrous oxide), the arterial and venous content of the tracer is plotted with time. The area between the arterial and venous curves provides the quantitative measurement of CBF. In addition, the arterial-venous difference in oxygen content may also be measured. This parameter can be used to calculate OEF and the metabolic rate for oxygen consumption $\left(\mathrm{CMRO}_{2}\right)$.

Received May 21, 2012; accepted after revision May 22.

From the Mallinckrodt Institute of Radiology (J.J.L., C.P.D.), Division of Comparative Medicine (C.B.F.), and Departments of Neurology and Neurological Surgery (C.P.D.), Washington University School of Medicine, St. Louis, Missouri; Department of Neurology (W.J.P.), University of North Carolina School of Medicine, Chapel Hill, North Carolina; and Department of Medicine (P.J.B.), University of New Mexico, Albuquerque, New Mexico.

This work was supported by NINDS P01 NS35966.

Please address correspondence to C.P. Derdeyn, MD, Mallinckrodt Institute of Radiology, Washington University of Medicine, 4525 Scott Ave, Box 8131, Suite 2218, St. Louis, Missouri, 63110; e-mail: derdeync@wustl.edu

- Indicates open access to non-subscribers at www.ajnr.org

http://dx.doi.org/10.3174/ajnr.A3270
The purpose of this Technical Note is to describe a system for the quantitative Kety-Schmidt measurement of CBF in a highfield MR imaging environment that could be applied to human or animal studies.

\section{TECHNIQUE}

\section{Animal Preparation}

This study used 2 male cynomolgus macaques, Macaca fascicularis, and 1 female baboon, Papio anubis. The baboon was ultimately preferred for its larger size, which facilitated percutaneous placement of lines.

\section{Vascular Interventions}

After fasting 12 hours, including 2 hours without water, and receiving atropine $50 \mu \mathrm{g} / \mathrm{kg} \mathrm{IM}$, the animal was brought to an interventional suite and given ketamine, $10-15 \mathrm{mg} / \mathrm{kg}$ IM. A softcuff endotracheal tube was placed, and general anesthesia was obtained with isoflurane vaporized into medical grade oxygen (Airgas Puritan Medical, St. Louis, Missouri) by using an MR imaging-compatible ventilator (Surgivet, Waukesha, Wiscon$\sin )$. Anesthesia delivery, blood pressure, $\mathrm{pCO}_{2}$, and oxygen saturation were monitored continuously (Surgivet). Following anesthesia induction, 22-ga angiocatheters (Terumo Medical, Elkton, Maryland) were percutaneously placed in the femoral artery and contralateral femoral vein. Both angiocatheters were exchanged for 4F catheters (Cook Medical, Bloomington, Indiana). The venous catheter typically required a J-wire to advance through the femoral sheath to the jugular bulb. Placement was verified under fluoroscopy (ioversol 68\%, Optiray; Mallinckrodt, St. Louis, Missouri). Unfractionated heparin was administered to 20-40 USP units/kg. 


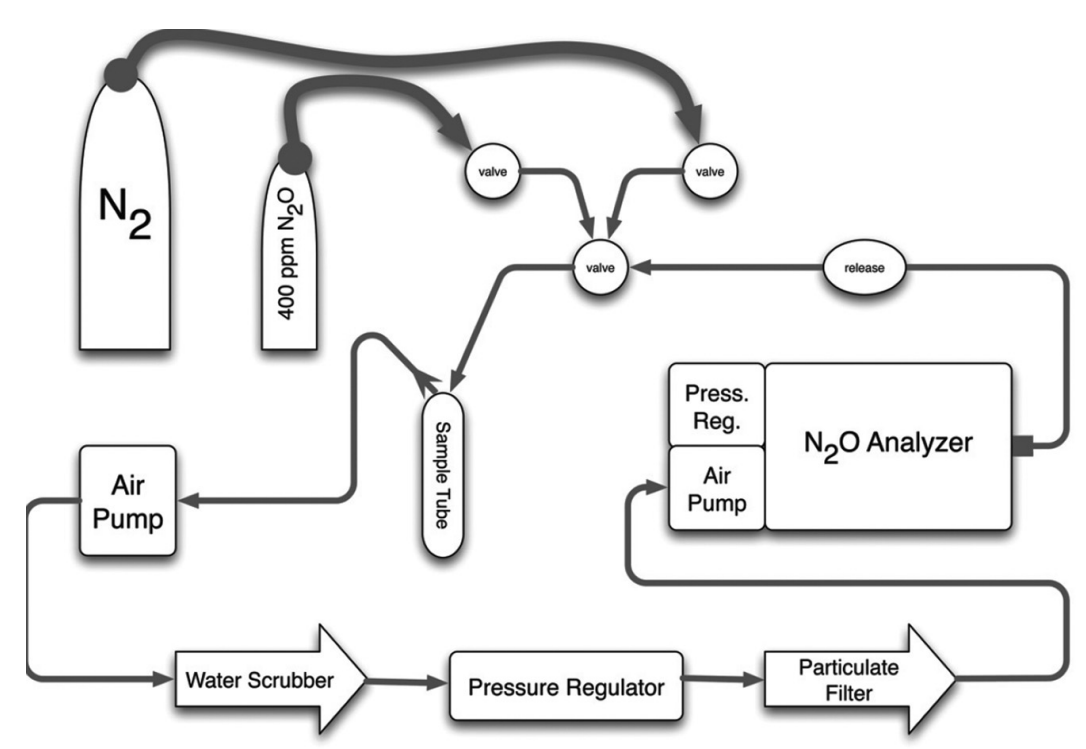

FIG 1. Nitrous oxide concentration for each timed blood sample is measured with an infrared gas-analyzer and sampling apparatus. Arrowheads indicate flow of gases. Valves introduce zero $\left(\mathrm{N}_{2}\right)$ and span $\left(\mathrm{N}_{2} \mathrm{O}\right)$ gases.

The animal was subsequently transported to the MR imaging suite. The animal rested quietly for a minimum of 15 minutes to attain physiologically steady conditions. At least 3 hours separated ketamine administration from hemodynamic measurements to minimize drug effects on CBF. Line placement was confirmed with magnetization-prepared rapid acquisition of gradient echo or MRA. To initiate Kety-Schmidt measurements, the animal was ventilated with $25 \%-45 \% \mathrm{~N}_{2} \mathrm{O}, 21 \% \mathrm{O}_{2}$, and $1-2.5 \%$ isoflurane, balanced with room air. Sequential arterial and venous samples were collected in 1-mL syringes (Becton Dickinson, Franklin Lakes, New Jersey) for a minimum of 20 minutes. Syringes were disconnected, held orifice-down, sealed with plastic Monoject Tip Caps (Tyco Healthcare Group, Mansfield, Massachusetts), and placed on ice. Primary standard nitrous oxide tracer was delivered via MR imaging-compatible cylinders (Airgas Puritan Medica, Randor, Pennsylvania). Syringes were attached to luer lok valves (MedEx, Monsey, New York), and approximately $0.5 \mathrm{~mL}$ of blood was extracted at 30-, 60-, or 120-second intervals. The time of extraction was annotated with MR imaging-compatible microphones and digital audio capture (High Criteria, Richmond Hill, Ontario, Canada) to a laptop computer. The dead space for the arterial catheter and valve was $0.2 \mathrm{~mL}$; for the venous catheter, it was $1.0 \mathrm{~mL}$; and for blood-collection syringes, it was $0.03 \mathrm{~mL}$.

At the conclusion of the procedures, all catheters were removed and hemostasis was obtained by vascular compression. The animal was allowed to recover from anesthesia while under continuing monitoring. All animal procedures were reviewed by the institutional review board and animal studies committees of Washington University School of Medicine.

\section{Nitrous Oxide Analysis}

Sampling syringes were analyzed for nitrous oxide content. Each syringe was removed from ice and shaken to settle blood products, and the blood volume was measured to $0.01-\mathrm{mL}$ accuracy. The syringe was held orifice-down, the tip cap was removed, an 18-ga needle (Becton, Dickenson and Company, Franklin Lakes, New Jersey) was attached, and the needle was inserted into a 10 -mL Vacutainer tube (Becton, Dickenson and Company) prefilled with desiccated sodium heparin. The blood sample was injected, the syringe was rinsed with unfractionated heparin, and the rinse was also injected into the Vacutainer. After mechanical mixing (VWR International, West Chester, Pennsylvania), the Vacutainer was returned to ice.

The nitrous oxide analysis apparatus is sketched in Fig 1. The circuit comprised Tygon tubing (Saint Gobain, Courbevoie, France), luer-lok valves, and mechanical components as drawn in Fig 1. The quantity of nitrous oxide gas present in each Vacutainer tube was measured with a NDIR nitrous oxide analyzer (Teledyne Analytical Instruments, City of Industry, California). Our analyzer unit was equipped with the following (all components from Liston Scientific, Irvine, California): a recirculation pump, wateradsorbing percolation filter, particulate filter, dual pressure regulators set to prevent exceeding the device tolerance of $206 \mathrm{~mm} \mathrm{Hg}$, and emergency valve releases. Zero deflection of the NDIR unit was calibrated with an ultrahigh purity compressed nitrogen (Airgas Puritan Medical). A dual-stage fitting regulator was used to introduce the "zero gas" to the analysis circuit. Full deflection of the NDIR unit was calibrated with a primary standard mixture comprising $400 \mathrm{ppm}(0.04 \pm 0.0004 \%)$ nitrous oxide balanced with ultrahigh purity nitrogen (Airgas Puritan Medical). A singlestage fitting regulator was used to connect the "span gas." The NDIR unit and the sampling circuit were calibrated with zero and span gases at regular intervals during processing to ensure acceptable instrument drift. Leakage and stability of the sampling circuit were also periodically tested with 400 -ppm nitrous oxide in the presence of actively pumped recirculation.

\section{Kety-Schmidt Calculations}

The presence of $\mathrm{H}_{2} \mathrm{O}$ or $\mathrm{CO}_{2}$ confounds measurements of $\mathrm{N}_{2} \mathrm{O}$ in the gas analyzer; only $\mathrm{H}_{2} \mathrm{O}$ was scrubbed. The sample chamber of the analyzer was specified to hold $77 \mathrm{~mL}$ of sample gas. By dilution of a known volume of primary standard 400-ppm nitrous oxide, the total volume of the apparatus was determined to be $333 \mathrm{~mL}$. The quantity of in vivo nitrous oxide was determined by back- 


\section{Arterial \& Venous N2O Wash-Out Curves}

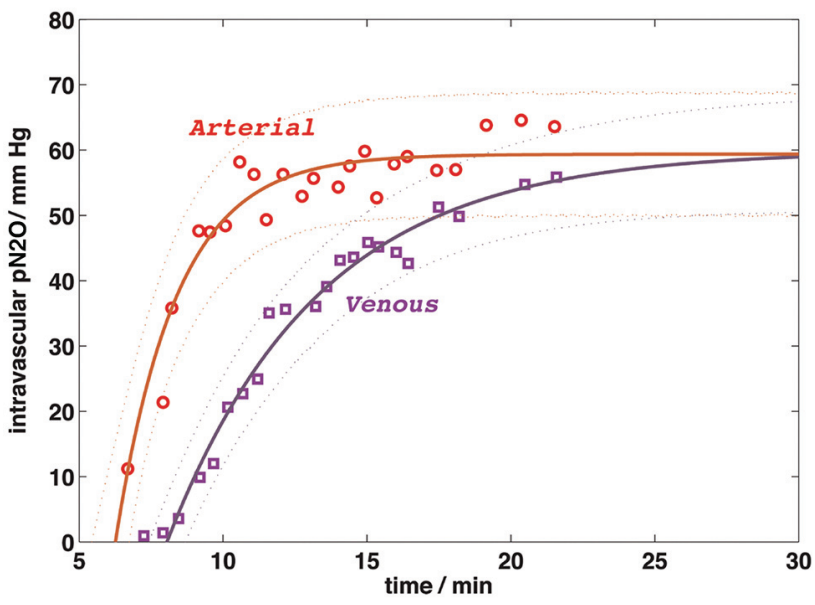

FIG 2. Nitrous oxide wash-in with arterial and venous catheters, respectively. Dead space corrections apply. Fitting of the Kety-Schmidt model, $p_{a} \mathrm{~N}_{2} \mathrm{O}=13.5+46\left[1-\exp (-0.47(t-6.81)]\right.$ and $p_{v} \mathrm{~N}_{2} \mathrm{O}=$ $7.55+52(1-\exp [-0.19(t-8.76)]$, is shown with $95 \%$ confidence intervals and residuals. The calculated global CBF is $52.5 \mathrm{~mL} / \mathrm{min} / 100 \mathrm{~g}$.

calculated serial dilutions and dead space contributions. An example of measurement of nitrous oxide is shown in Fig 2.

Dead space was modeled by using conservation of volumes and mass. The nitrous oxide concentration in vivo, $c_{m}$, for the $m$ th sampling of volume $V m$ was expressed as

$$
\begin{gathered}
c_{1}=N_{s, 1} / V_{1} \\
c_{m}=N_{s, m}+g_{m} g_{m^{-1}} V_{d} c_{m^{-1}} /\left(V_{m}+g_{m} V_{d}\right), m>2 \\
g_{m}=\bmod \left(V_{m}, V_{d}\right) / V_{d} .
\end{gathered}
$$

The measured quantity of nitrous oxide in the $m$ th sample is $N_{m}$. The dead space is $V_{d}$. The modulus is denoted by mod.

The cerebral uptake of $\mathrm{N}_{2} \mathrm{O}$ at time $t, q(t)$, is proportional to both $\mathrm{CBF}$ and the gradient of $\mathrm{N}_{2} \mathrm{O}$ concentrations between arteries, $C_{A}$, and veins, $C_{V}$ : The change in time of the quantity of nitrous oxide in an organ, $q(t)$, is proportional to the arterial flow, $F$; the gradient of nitrous oxide concentrations between feeding arteries, $C_{A}(t)$; and draining veins, $C_{V}(t)$, as described by the Fick equation. ${ }^{4}$ Integration over the experiment time yields the flow:

$$
F=\frac{q(T)}{\int_{0}^{T}\left[C_{A}(t)-C_{V}(t)\right] d t} .
$$

The uptake of nitrous oxide, $q(T)$, is not directly measurable but can be estimated from the steady-state nitrous oxide concentration in venous output weighted with a blood-brain partition coefficient, empirically determined to be 1.0 in primates, and a blood-gas partition coefficient. ${ }^{5}$

\section{DISCUSSION}

There are several aspects of this technique related to arterial and venous sampling that may impact both the accuracy of the results and the success of the procedure. The tip of the venous catheter should be near the jugular foramen and not selectively into an external vein. If venous blood is contaminated from facial or other external veins, the measured nitrous content will not reflect the cerebral washout only. This effect is minimal, given the relatively small contribution of external venous drainage compared with the cerebral circulation. Samples can also be confounded by significant dead space effects. On the arterial side, repeated access to the common femoral artery in small animals may be challenging. Cut-down procedures can impede future vascular access.

While our study demonstrates the feasibility of this technique in an MR imaging environment, several limitations should be noted. First, the method only provides global, not regional, data. To use this technique for validation purposes, it would be useful to make multiple measurements with different global CBF values. This could be accomplished by changing $\mathrm{CBF}$ through changes in $\mathrm{pCO}_{2}$. These changes in $\mathrm{CBF}$ would lead to changes in OEF as well. Oxygen metabolism can be changed pharmacologically. Second, the technique requires 15-20 minutes per measurement, allowing achievment of steady-state equilibrium of the tracer and subsequent washout. For simultaneous measurements by using KetySchmidt and MR imaging acquisitions, care would be required to maintain all the physiologic variables during the time of acquisition.

This preparation will also provide measurements of global oxygen extraction and oxygen consumption. This requires measurement of arterial $\left(\mathrm{CaO}_{2}\right)$ and venous oxygen content $\left(\mathrm{CvO}_{2}\right)$. Once these values are known, the arterial-venous difference for oxygen content $\left(\mathrm{AVDO}_{2}\right)$ is calculated by subtraction $\left(\mathrm{CaO}_{2}-\mathrm{CvO}_{2}\right)$. OEF is equal to the $\mathrm{AVDO}_{2}$ divided by the $\mathrm{CaO}_{2} \cdot \mathrm{CMRO}_{2}$, the metabolic rate of oxygen consumption, is equal to $\mathrm{CBF}$ (the delivery of oxygen) times the OEF (the fraction of oxygen removed) times $\mathrm{CaO}_{2}$ (the amount of oxygen in the arterial blood).

In summary, we present a simple method for adapting the KetySchmidt technique for quantitative CBF measurement in an MR imaging environment. This system may also be used for the measurement of arterial-venous oxygen difference, which in turn may be used to calculate whole-brain oxygen extraction and the metabolic rate of oxygen consumption. This technique may have value in the validation of MR imaging methods of measurement of CBF, oxygen extraction, and the metabolic rate of oxygen consumption in humans or other animals.

Disclosures: Colin Derdeyn—UNRELATED: Consultancy: W.L. Gore and Associates, Comments: medical device company, products not related to this subject, Expert Testimony: brain aneurysm and acute ischemic stroke treatment cases, not related to this subject, Stock/Stock Options: nFocus, Pulse Therapeutics, Comments: brain aneurysm and acute stroke treatment companies (neither related to this topic).

\section{REFERENCES}

1. Ostergaard L, Smith DF, Vestergaard-Poulsen P, et al. Absolute cerebral blood flow and blood volume measured by magnetic resonance imaging bolus tracking: comparison with positron emission tomography values. J Cereb Blood Flow Metab 1998;18:425-32

2. Lin W, Celik A, Derdeyn C, et al. Quantitative measurements of cerebral blood flow in patients with unilateral carotid artery occlusion: a PET and MR study. J Magn Reson Imaging 2001;14:659-67

3. Kety SS, Schmidt CF. The determination of cerebral blood flow in man by the use of nitrous oxide in low concentrations. Am J Physiol $1945 ; 143: 53$

4. Boyle PJ, Nagy RJ, O'Connor AM, et al. Adaptation in brain glucose uptake following recurrent hypoglycemia. Proc Natl Acad Sci U S A 1994;91:9352-56

5. Kety SS. The theory and applications of the exchange of inert gas at the lungs and tissues. Pharmacol Rev 1951;3:1-41 